\title{
BMJ Open Patient-centred and family-centred care of critically ill patients who are potential organ donors: a qualitative study protocol of family member perspectives
}

To cite: Zheng K, Sutherland S, Cardinal $\mathrm{P}$, et al. Patientcentred and family-centred care of critically ill patients who are potential organ donors: a qualitative study protocol of family member perspectives. BMJ Open 2020;10:e037527. doi:10.1136/ bmjopen-2020-037527

- Prepublication history for this paper is available online. To view these files, please visit the journal online(http://dx.doi. org/10.1136/bmjopen-2020037527).

Received 06 February 2020 Revised 06 March 2020 Accepted 19 April 2020
Check for updates

(C) Author(s) (or their employer(s)) 2020. Re-use permitted under CC BY-NC. No commercial re-use. See rights and permissions. Published by BMJ.

For numbered affiliations see end of article.

Correspondence to

Dr Aimee J Sarti; asarti@toh.ca

Katina Zheng (D) , ${ }^{1}$ Stephanie Sutherland, ${ }^{2}$ Pierre Cardinal, ${ }^{2}$ Maureen Meade, ${ }^{3}$ Angele Landriault, ${ }^{4}$ Brandi Vanderspank-Wright, ${ }^{5,6}$ Sabira Valiani, ${ }^{7}$ Sam Shemie, ${ }^{8,9}$ Amber Appleby, ${ }^{9}$ Sean Keenan, ${ }^{10,11}$ Matthew Weiss, ${ }^{12}$ Kim Werestiuk, ${ }^{13}$ Andreas H Kramer, ${ }^{14}$ Joann Kawchuk, ${ }^{7}$ Stephen Beed, ${ }^{15}$ Sonny Dhanani, ${ }^{16}$ Giuseppe Pagliarello, ${ }^{2}$ Michaël Chasse, ${ }^{17}$ Ken Lotherington, ${ }^{9}$ Mary Gatien, ${ }^{18}$ Kim Parsons, ${ }^{19}$ Jennifer Chandler, ${ }^{20}$ Peter Nickerson, ${ }^{21}$ Jim Kutsogiannis, ${ }^{22}$ Aimee J Sarti ${ }^{2,6}$

\section{ABSTRACT}

Introduction In a patient-centred and family-centred approach to organ donation, compassion is paramount. Recent guidelines have called for more research, interventions and approaches aimed at improving and supporting the families of critically ill patients. The objective of this study is to help translate patient-centred and family-centred care into practice in deceased organ donation.

Methods and analysis This will be a national, qualitative study of family members of deceased organ donors in Canada. We will include family members who had been approached regarding an organ donation decision, including those who agreed and declined, at least 2 months and no later than 3 years after the patients' death. Data collection and analysis is ongoing and will continue until September 2020 to include approximately 250 participants. Family members will be identified and recruited from provincial organ donation organisation databases. Four experienced qualitative researchers will conduct telephone interviews in English or French with audio-recording for subsequent transcription. The research team will develop a codebook iteratively through this process using inductive methods, thus generating themes directly from the dataset.

Ethics and dissemination Local research ethics boards (REB) at all participating sites across Canada have approved this protocol. The main REB involved is the Ottawa Health Science Network REB. Data collection began in August 2018. Publication of results is anticipated in 2021. Study findings will help improve healthcare provider competency in caring for potential organ donors and their families and improve organ donation consent rates. Findings will also help with the development of educational materials for a competency-based curriculum for critical care residents.

\section{Strengths and limitations of this study}

This will be the largest Canadian cohort of substitute decision-maker perspectives on organ donation to date; all but one Canadian province are represented (the province of Prince Edward Island and the Canadian territories (Nunavut, Northwest Territories and Yukon) were not included as there are no organ donation organisations in those regions).

- Perspectives of families who consented to or declined organ donation following both cardiocirculatory declaration of death and neurological declaration of death will be explored in this study.

- Results will help to align competency-based curricula for healthcare professionals with the lived experiences and perspectives of the families of organ donors.

- The study will identify problems and propose solutions that are context-specific, reflecting the many different situations experienced by patients and families.

- Because interviews are limited to English and French languages, the perspectives of Canadian indigenous and immigrant populations are likely to be underrepresented, as well as those of family members who had declined organ donation.

\section{INTRODUCTION}

Patient-centred and family-centred care (PFCC) is an approach to healthcare that is respectful of and responsive to individual patients and families' needs and values. ${ }^{1}$ This approach has been recognised by many jurisdictions as a priority in healthcare service delivery. $^{2}$ 
Conceptually, PFCC is a model in which healthcare providers are encouraged to partner with patients or their family (if patients are incapable of participating) to co-design and deliver high-quality, personalised care that provides patients and families with the high-quality care they deserve. 'Family-centred care' is a respectful and responsive approach to healthcare that meets the needs and values of individual families and is mainly characterised by: family presence in the intensive care unit (ICU), family support and communication with family members. ${ }^{1-3}$

The most recent PFCC guidelines are the result of a new and more rigorous analysis of evidence concerning the role of families in ICU care. ${ }^{1}$ These guidelines represent the current state of international science in familycentred care and family support for family members of critically ill patients. However, to date, there is limited empirical evidence to guide clinicians on how best to practice in this manner. ${ }^{1}$ For frontline healthcare providers, who interact directly with patients and families, the implementation of PFCC goes beyond general principles to include pragmatic and actionable items that can be introduced into every-day practice to support increasingly patient-centred/family-centred behaviour. ${ }^{45}$

Many different situations may be experienced by patients and families related to organ donation during this high-stress time; hence, we anticipate that the stated problems and solutions will be context-specific. For example, the needs of a family whose loved one has just been admitted to the emergency department will differ from their needs when their loved one, having just been declared deceased by circulatory criteria, is being transported to the operating room for organ recovery. Therefore, a major objective of this work is to list and describe the situations, identified by family members, that demand different action plans and/or communication strategies. Expert clinicians rely heavily on pattern recognition in patient care. They learn to recognise situations, often through trial and error, that demand a change in their standard approach. ${ }^{6} 7$ Unfortunately, given the scarcity of organ donation, even experts may not be aware of all the emotionally charged situations that may traumatise families. Positive and negative psychological effects of donation are reported by families and it is critical to understand better how to navigate the diversity of family experience in multicultural societies to capture the psychological benefits for families and avoid the factors that may result in harm. ${ }^{8-11}$ We believe that this study will comprehensively identify situations in which families are most vulnerable, enabling healthcare providers to introduce specific interventions to alleviate their suffering. Furthermore, this study will help identify the particular needs of provinces and regions (with different policies and resources) and populations (eg, families who have agreed to donation, agreed to donation but donation was not possible, or refused donation).

To improve PFCC in organ donation, Canadian Blood Services (CBS) funded a nation-wide qualitative study to comprehensively explore the donation process for both paediatric and adult patients from the perspectives of family members. Interviews are being conducted with family members directly involved in the decision-making surrounding donation, to identify the problems they encountered and the solutions they propose. We then plan to use this qualitative data to develop educational interventions targeting healthcare providers who care for potential donors and their families.

This work will be the largest, Canadian qualitative study to date and will provide the necessary information required to develop a genuine national patient-centred and family-centred approach to donation. We will describe the current donation experiences of Canadian families, including challenges and successes, and determine opportunities for improvement at the policy and practice levels.

\section{Aims}

Describe the families' complete journey through the processes of organ donation to better understand their experience and inform the development and implementation of best clinical practices patient and family centred.

\section{METHODS AND ANALYSIS}

\section{Study design}

This is a retrospective, qualitative study using telephone interviews with family members who were approached for organ donation in the ICU in the last 3 years. Family members include anyone primarily responsible to decide on behalf of patients, regardless of whether they are biologically related. This study is being conducted following the consolidated criteria for reporting qualitative research (COREQ).

\section{Setting and context}

Participants will be enrolled from all Canadian provinces with organ donation organisations (ODOs), which therefore excludes Prince Edward Island and the territories.

\section{Eligibility criteria}

We will include all consenting family members, 18 years old or older, who had been asked to consider paediatric or adult organ donation at a Canadian ICU, between 2 months and 3 years after the death of their loved one, whether or not they ultimately consented to, or declined organ donation. This includes both donations following a neurological determination of death (NDD) or donation after circulatory death (DCD). Family members who cannot understand or interpret interview questions either because of language (non-English or non-French speaking) or cognitive barriers will be excluded.

\section{Organ donation database}

Each ODO will compile a database of eligible family members, including name, phone number, address, age, sex, approach for NDD or DCD, donation decision and their relationship with the patient. The ODO will initially 
reach out to the family members to see if they are interested in participating and if they are agreeable to be contacted by the research team. For family members who agree to be contacted by the research team, the following patient information including the initial diagnosis, date of death, age and sex will be collected. For family members who decline to be contacted, the same data will be collected, in aggregate form, to determine whether consenting family members are representative of the whole group. The ODOs will compile and keep the complete database of contacted family members. The research team will only gain access to the complete database once recruitment is completed, at which time, a de-identified database will be released without names, addresses and phone numbers. Each family member who agrees to be contacted will be assigned a unique study number.

\section{Recruitment}

Family members will be recruited using a streamlined process (figure 1). Potential participants will be identified retrospectively through provincial ODO databases. When possible, information letters will be mailed to family members to inform them of the study. Approximately 2 weeks later, provincial ODO coordinators will attempt to contact them by phone (minimum of two attempts) to explain how their contact information was obtained and why. Only family members who agree to be contacted by the research team will be contacted for interviews. The research team will then call family members to discuss the study and to organise a telephone interview if consent is obtained.

\section{Sample size}

We estimate that a sample of approximately 250 family members will provide sufficient data to identify all the situations in which patients and families are most vulnerable, including variations based on patient and family characteristics as well as different time points in their journey. Characteristics include: consented and donated (NDD and DCD), consented and not able to donate (NDD and DCD), consented and changed mind (NDD and DCD), declined (NDD and DCD), medical assistance in dying (MAID), adult and paediatric (NDD and DCD). This sample size will also allow an exploration for regional differences across the provinces. Ultimately, the sample size will be determined through iterative data collection and analyses, with recruitment ending when no new themes emerge. ${ }^{12}$

\section{Interview guide development}

We will employ a semi-structured interview guide, designed to follow a broad, predetermined line of inquiry that is flexible and can evolve as data collection unfolds, permitting exploration of emerging themes. The interview guide was created by an interdisciplinary team of investigators with expertise in critical care, palliative care, organ donation, medical education and sociological and qualitative research methods. The guide builds on prior

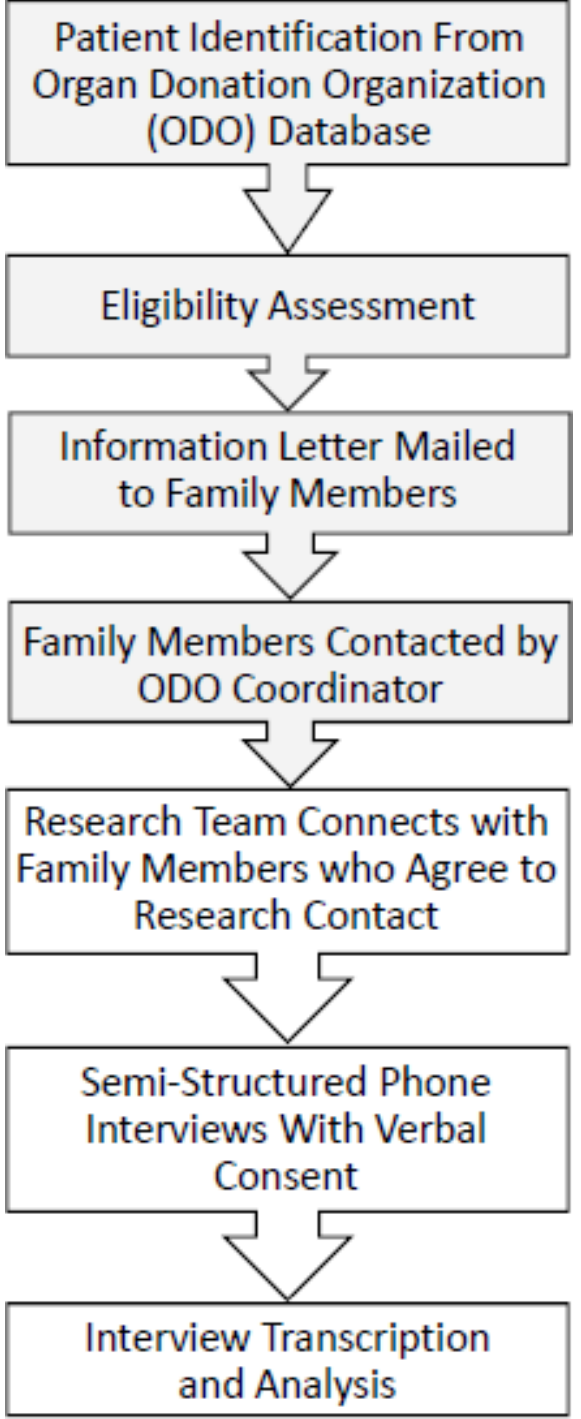

Figure 1 Study flow. The process of family member enrolment following organ donor patient identification from the organ donation organisation database. Note that this process will be adapted in some provinces due to internal policies.

work done for a provincial study on organ donation in Ontario, Canada. ${ }^{13}$ The guide was further presented to a committee of family members, members of the Canadian Critical Care Trials Group and organ donation leaders representing every ODO in Canada and further revised based on this feedback.

The content of the interview guide includes a series of open-ended questions; participants will be guided to discuss their experience starting when they first learnt that their loved one was critically ill, through their in-hospital experience and finally, their posthospital bereavement. Interviews will explore the communication about the critical illness and donation, as well as the care and support provided during that time. Family members will be specifically asked their perspectives on the provision of PFCC and the ways in which care could have been enhanced and improved. This flexible and evolving approach will 
help capture the full richness of the participant experience while allowing for the exploration of emerging themes. The interview guide was translated into French and content reviewed by French-speaking members of the research team and Transplant Québec. The interview guide was pilot tested with research team members and with participants of the family member committee.

\section{Data collection}

Family members, who agree to be contacted, will be scheduled for one-on-one telephone interviews, lasting approximately $45-60 \mathrm{~min}$. Interviews will be conducted by several highly trained and qualified researchers (AS, $\mathrm{SSu}, \mathrm{BVW}, \mathrm{AL}$ ). Two of the researchers are fluently bilingual and able to conduct interviews in both English and French (AL, BVW). AS is an Intensivist at the Ottawa Hospital with a subspecialty in Palliative Care Medicine. AS has over 10 years of experience in conducting qualitative research and recently led a provincial needs assessment which included interviewing family members. SSu, $\mathrm{PhD}$, has over 20 years of experience in conducting and analysing qualitative research and has taught graduate and continuing medical education classes on conducting rigorous high-quality qualitative research. $\mathrm{BVW}, \mathrm{PhD}$, has over 15 years of experience as a qualitative researcher and maintains Canadian Nurses Association Certification in adult critical care. AL is a project manager and qualitative researcher with extensive experience in working on largescale mixed-method and qualitative studies. Data collection started in August 2018 and is ongoing.

\section{Analyses of the results}

Interviews will be audio recorded and transcribed verbatim. All data will be verified for accuracy and entered into ATLAS.ti software (Scientific Software Development $\mathrm{GmbH}$, Berlin, Germany), a qualitative research software programme to facilitate data management and analysis. The analysis team will consist of five researchers who will collaboratively participate in coding meetings so as to develop the coding tree and codebook (AS, SSu, $\mathrm{AL}, \mathrm{BVW}$ and SV). Inductive coding techniques will be applied, with codes generated directly from the data sets. ${ }^{14}$ Ongoing group discussions will be used to record new and emergent codes. All members of the qualitative data analysis team will participate in the development of the codebook. The same initial interview transcripts will be independently coded by all five team members and then discussed in coding meeting to identify emergent codes and reach consensus on the coding tree and codebook. As multiple coders are required to independently code the transcripts, once the codebook is established, intercoder reliability will be assessed. ${ }^{15}$ ATLAS.ti Software will be used to assess intercoder reliability. The team will continue to code as a group until a Krippendorff alpha of at least 0.85 is achieved, at which point independent coding will commence. ${ }^{16}$ Data collection and analysis will be conducted iteratively until thematic saturation is achieved.

\section{Patient and public involvement}

The research question and design were informed by important feedback received from patient families during our previous provincial study. ${ }^{13}$ Input from our family member committee, established specifically for this study, was invaluable to the creation of our interview tools. They will continue to be actively involved in reviewing and advising in our study findings.

\section{ETHICS AND DISSEMINATION \\ Ethical approval}

This national study has been approved by local institutional research ethics boards (REBs) across Canada. The REBs involved are the Ottawa Health Science Network REB, Ontario, the Conjoint Health Research Ethics Board (CHREB), the University of Calgary, Alberta, the University of British Columbia Office of Research Services Behavioural REB, British Columbia, the University of Manitoba HREB, Manitoba, the Horizon Health Network REB, New Brunswick, Canada, Newfoundland and Labrador HREB, Newfoundland, the Nova Scotia Health Authority (NSHA) REB, Nova Scotia, the Bureau d'éthique de la recherche du CHU de Québec-Université Laval, Quebec, and the University of Saskatchewan Behavioural REB (Bet-REB), Saskatchewan.

There is no relationship between family members and the person obtaining consent. The research study does not include individuals incapable of giving informed consent or under the age of 18 years. The research project does not involve situations where consent cannot be obtained.

\section{Consent}

The information letter sent to the family members will include a copy of the consent form. The research team will provide family members who accept to be contacted with information on the study aims, objectives, methods, funding and investigators' contact information and affiliations. Verbal consent will be obtained from family members over the phone. They will be informed that they have the right to decline or withdraw at any time without reprisal. They will also be informed that there will be no further contact with research members should they decide that they no longer wish to participate at any time after consenting.

\section{Information security}

After each interview, audio recordings will be immediately and securely transferred from the audio recording device to an encrypted central hospital drive at The Ottawa Hospital and deleted from the audio recorder. Shared files remain in Canada and are fully encrypted during transmission. Audio recordings of individual interviews will be de-identified at the time of transcription. All digital recordings of interviews will be kept for the duration of this project on a secure server at the hospital and will then be destroyed. Digital audio files are required as interviews are transcribed for analysis. They 
are kept to verify the accuracy of the transcription. All study records will be stored for 10 years and then deleted and/or shredded.

\section{Outcome and dissemination}

This study has been designed to inform the development of an organ donation competency-based curriculum. The curriculum will be family centred, incorporating their recommendations, and aligned with the standards and expectations of the Royal College of Physicians and Surgeons of Canada, such that the programme can be disseminated and adopted by the Adult and Pediatric Critical Care Medicine Subspecialty programmes nationwide. In addition, multiple strategies will be used to ensure that findings can be shared effectively with academic, public and policy audiences. Findings from this study will be submitted for peer review in an appropriate journal and presented at academic conferences. Publication of results is anticipated in 2021. A website will be developed by CBS to share the results of this study, both with the public and healthcare professionals.

\section{STRENGTHS AND LIMITATIONS OF THE STUDY \\ Strengths}

In this study, a broad range of perspectives of families will be explored, including those who consented to or declined organ donation following both cardiocirculatory declaration of death and neurological declaration of death. This will be the largest Canadian cohort of family members perspectives and experiences who have had to make an organ donation decision to date. The results of this study will identify problems and solutions proposed by families that are context-specific, reflecting many of the different situations experienced by patients and families. Hence, findings will help those hoping to translate PFCC into practice.

\section{Limitations}

There are several limitations to this study. Due to the inherent characteristics of the study subjects, the proportion of families who agree to be interviewed may be low, potentially introducing a selection bias. Additionally, because interviews could only be conducted in English and French, groups who do not speak the two official languages may be under-represented, including some indigenous Canadians and immigrant families. We may also miss perspectives from some remote communities because the Canadian Territories could not be included in this study. Finally, as the timing of interviews can range from 2 months to 3 years after the donation decision, there is a potential for recollection bias from interviewees, whose perspectives may change over time. However, this timeframe was chosen to answer an empirical question that persists in the donation and transplantation literature. Namely, what is the optimal time to contact a grieving family member for an interview? After conducting a literature review on the topic (manuscript in progress), we found that currently there is significant variation in the time a family member is approached for an interview, for example, studies who approached families within the 0-3 months timeframe, ${ }^{17-20}$ studies that approached families within a 1-year timeframe, ${ }^{21-23}$ and at the other end of the spectrum studies that included families in the 1-3years timeframe. ${ }^{24-26}$ Families are also asked near the end of the interview what they feel is the most appropriate timeframe to conduct an interview.

\section{Author affiliations}

${ }^{1}$ University of Ottawa, Faculty of Medicine, Ottawa, Ontario, Canada

${ }^{2}$ Department of Critical Care, Ottawa Hospital, Ottawa, Ontario, Canada

${ }^{3}$ Department of Critical Care, Hamilton Health Sciences, Hamilton, Ontario, Canada

${ }^{4}$ Practice, Performance and Innovation (PPI) Unit, Royal College of Physicians and

Surgeons of Canada, Ottawa, Ontario, Canada

${ }^{5}$ School of Nursing, University of Ottawa Faculty of Health Sciences, Ottawa,

Ontario, Canada

${ }^{6}$ Ottawa Hospital Research Institute, Ottawa, Ontario, Canada

${ }^{7}$ Department of Critical Care, Saskatchewan Health Authority, Saskatoon,

Saskatchewan, Canada

${ }^{8}$ Pediatrics, McGill University, Montreal, Quebec, Canada

${ }^{9}$ Canadian Blood Services, Ottawa, Ontario, Canada

${ }^{10} \mathrm{BC}$ Transplant, Vancouver, British Columbia, Canada

${ }^{11}$ Division of Critical Care, UBC Department of Medicine, Vancouver, British

Columbia, Canada

${ }^{12}$ Population Health and Optimal Health Practices Research Unit, Trauma-

Emergency-Critical Care Medicine, CHU de Quebec-Universite Laval, Quebec city,

Quebec, Canada

${ }^{13}$ Transplant Manitoba, Winnipeg, Manitoba, Canada

${ }^{14}$ University of Calgary, Calgary, Alberta, Canada

${ }^{15}$ Department of Critical Care, Dalhousie University Faculty of Medicine, Halifax, Nova Scotia, Canada

${ }^{16}$ Critical Care, Children's Hospital of Eastern Ontario, Ottawa, Ontario, Canada ${ }^{17}$ Department of Critical Care Medicine, Centre Hospitalier de L'Universite de Montreal, Montréal, Quebec, Canada

${ }^{18}$ Horizon Health Network, Miramichi, New Brunswick, Canada

${ }^{19}$ Organ Procurement and Exchange of Newfoundland and Labrador (OPEN), St. John's, Newfoundland and Labrador, Canada

${ }^{20}$ University of Ottawa Faculty of Law Common Law Section, Ottawa, Ontario, Canada

${ }^{21}$ University of Manitoba Faculty of Health Sciences, Winnipeg, Manitoba, Canada

${ }^{22}$ Department of Critical Care Medicine, University of Alberta Faculty of Medicine and Dentistry, Edmonton, Alberta, Canada

Acknowledgments We would like to extend our gratitude to all the families who are giving their time to participate in this study and share their experiences. We would also like to thank the organ donation organisations involved, including BC Transplant, Transplant Manitoba Gift of Life Program, the New Brunswick Organ \& Tissue Donation Program, the Critical Care Organ Donation Program (Nova Scotia), the Organ Procurement Exchange of Newfoundland and Labrador (OPEN), the Trillium Gift of Life Network (TGLN), Transplant Quebec, Saskatchewan Donation and Transplant Programs, Southern Alberta Organ and Tissue Donation Program, and the Human Organ Procurement and Exchange Program (HOPE). Finally, we are grateful to the members of our family member committee for their time and contribution to the study.

Contributors All authors have made substantial contributions to the conception of this work. AJS, SSu, PC, AA, SSh and KL conceived the research idea and study design. KZ wrote the initial draft of this manuscript. KZ, SSu, PC, MM, AL, BVW, SV, SSh, AA, SK, MW, KW, AHK, JKa, SB, SD, GP, MC, KL, MG, KP, JC, PN, JKu and AJS all edited and critically revised the research protocol and paper. All authors approved the final version of this manuscript.

Funding This study was supported by the Canadian Blood Services.

Competing interests None declared.

Patient consent for publication Not required.

Provenance and peer review Not commissioned; externally peer reviewed. 
Open access This is an open access article distributed in accordance with the Creative Commons Attribution Non Commercial (CC BY-NC 4.0) license, which permits others to distribute, remix, adapt, build upon this work non-commercially, and license their derivative works on different terms, provided the original work is properly cited, appropriate credit is given, any changes made indicated, and the use is non-commercial. See: http://creativecommons.org/licenses/by-nc/4.0/.

ORCID iD

Katina Zheng http://orcid.org/0000-0003-3004-156X

\section{REFERENCES}

1 Davidson JE, Aslakson RA, Long AC, et al. Guidelines for familycentered care in the neonatal, pediatric, and adult ICU. Crit Care Med 2017;45:103-28.

2 Bocci MG, Prestifilippo A, D'Alò C, et al. Family-centered care to improve family consent for organ donation. In: Organ donation and transplantation - Current status and future challenges. InTech, 2018: 1-13.

3 Seaman JB, Arnold RM, Scheunemann LP, et al. An integrated framework for effective and efficient communication with families in the adult intensive care unit. Ann Am Thorac Soc 2017;14:1015-20.

4 Dwamena F, Holmes-Rovner M, Gaulden CM, et al. Interventions for providers to promote a patient-centred approach in clinical consultations. Cochrane Database Syst Rev 2012;2012;48.

5 Santana MJ, Manalili K, Jolley RJ, et al. How to practice person-centred care: a conceptual framework. Health Expect 2018;21:429-40.

6 Kahneman D. Thinking, fast and slow. New York (NY: Farrar, Straus, Giroux Press, 2011.

7 Klein GA. Sources of power: how people make decisions. Vol 1. Cambridge (Mass: MIT Press, 1998.

8 Manzari ZS, Mohammadi E, Heydari A, et al. Exploring families' experiences of an organ donation Request after brain death. Nurs Ethics 2012;19:654-65.

9 Bellali T, Papadatou D. Parental grief following the brain death of a child: does consent or refusal to organ donation affect their grief? Death Stud 2006;30:883-917.

10 Tong Y-F, Holroyd EA, Cheng B. Needs and experiences of Hong Kong Chinese cadaveric organ donor families. Hong Kong J Nephrol 2006;8:24-32.

11 Weiss MJ, English SW, D'Aragon F, et al. Survey of Canadian intensivists on physician non-referral and family override of deceased organ donation. Can J Anesth 2019:313-23.
12 Creswell JW, Plano Clark VL. Best practices for mixed methods research. 2nd edn. Los Angeles: SAGE Publications, 2011.

13 Sarti AJ, Sutherland S, Healey A, et al. A multicenter qualitative investigation of the experiences and perspectives of substitute decision makers who underwent organ donation decisions. Prog Transplant 2018;28:343-8.

14 Creswell JW. Qualitative inquiry and research design: choosing among five approaches. 2nd edn. SAGE Publications, 2007.

15 MacPhail C, Khoza N, Abler L, et al. Process guidelines for establishing Intercoder reliability in qualitative studies. Qual Res 2016;16:198-212.

16 Krippendorff K. Computing Krippendorff's alpha-reliability part of the communication commons 2011.

17 Rodrigue JR, Cornell DL, Howard RJ. The instability of organ donation decisions by next-of-kin and factors that predict it. $\mathrm{Am} \mathrm{J}$ Transplant 2008;8:2661-7.

18 Rodrigue JR, Cornell DL, Krouse J, et al. Family initiated discussions about organ donation at the time of death. Clin Transplant 2010;24:493-9.

19 Marck CH, Neate SL, Skinner M, et al. Potential donor families' experiences of organ and tissue donation-related communication, processes and outcome. Anaesth Intensive Care 2016;44:99-106.

20 Neate SL, Marck CH, Skinner M, et al. Understanding Australian families' organ donation decisions. Anaesth Intensive Care 2015;43:42-50.

21 López Martínez JS, Martín López MJ, Scandroglio B, Martínez JSL, López MJM, et al. Family perception of the process of organ donation. qualitative psychosocial analysis of the subjective interpretation of donor and nondonor families. Span J Psychol 2008;11:125-36.

22 Kesselring A, Kainz M, Kiss A. Traumatic memories of relatives regarding brain death, Request for organ donation and interactions with professionals in the ICU. Am J Transplant 2007;7:211-7.

23 Jacoby L, Jaccard J. Perceived support among families deciding about organ donation for their loved ones: donor vs nondonor next of kin. Am J Crit Care 2010;19:e52-61.

24 Ashkenazi T, Cohen J. Interactions between health care personnel and parents approached for organ and/or tissue donation: influences on parents' adjustment to loss. Prog Transplant 2015;25:124-30.

25 Merchant SJ, Yoshida EM, Lee TK, et al. Exploring the psychological effects of deceased organ donation on the families of the organ donors. Clin Transplant 2008;22:341-7.

26 Taylor LJ, Buffington A, Scalea JR, et al. Harms of unsuccessful donation after circulatory death: an exploratory study. $A m \mathrm{~J}$ Transplant 2018;18:402-9. 